\title{
Surface plasmon resonance tuning of Ag nanoisland films using a $\mathrm{CO}$ laser
}

\section{$\operatorname{AUTHOR}(\mathrm{S})$ :}

Faniayeu, Ihar; Ishimatsu, Yuki; Nakajima, Takashi

\section{CITATION:}

Faniayeu, Ihar ...[et al]. Surface plasmon resonance tuning of Ag nanoisland films using a CO laser. Journal of Physics D: Applied Physics 2019, 52(29): 295103.

ISSUE DATE:

2019-07

URL:

http://hdl.handle.net/2433/259208

\section{RIGHT:}

This is the Accepted Manuscript version of an article accepted for publication in Journal of Physics D: Applied Physics. IOP Publishing Ltd is not responsible for any errors or omissions in this version of the manuscript or any version derived from it. The Version of Record is available online at 10.1088/1361-6463/ab1b7b.; This is not the published version. Please cite only the published version.; この論文は出版社版でありません。引用の際には出版社版をご確認ご利用くだ 


\title{
Surface plasmon resonance tuning of $\mathrm{Ag}$ nanoisland films using a $\mathrm{CO}_{2}$ laser
}

\author{
Ihar Faniayeu, Yuki Ishimatsu, and Takashi Nakajima \\ Institute of Advanced Energy, Kyoto University Gokasho, Uji, Kyoto 611-0011, Japan \\ E-mail: nakajima@iae.kyoto-u.ac.jp \\ January 2019
}

\begin{abstract}
We demonstrate that the surface plasmon resonance of $\mathrm{Ag}$ nanoislands (NIs) can be conveniently tuned through the laser power and irradiation time. For instance, the resonance wavelength of Ag NIs can be tuned from $548 \mathrm{~nm}$ to $452 \mathrm{~nm}$ under the $3 \mathrm{~W}$ laser power simply by varying the irradiation time from 5 sec to $2 \mathrm{~min}$. Interestingly, the position-dependent optical absorption spectra and SEM analysis reveals that the $\mathrm{Ag}$ NIs gradually disappear from the irradiation center even at $3 \mathrm{~W}$ if the irradiation time is longer. This means that the macroscopic diffusion of $\mathrm{Ag}$ atoms on the substrate takes place during the $\mathrm{CO}_{2}$ laser irradiation, and its driving force would be the temperature gradient along the radial direction with respect to the irradiation center due to the spatial beam profile of the laser.
\end{abstract}

\section{Introduction}

Metallic nanostructures are actively used for the manipulation of light, and indeed they have proved their usefulness for biosensors, photovoltaic devices, energy harvesting, plasmonics, etc. $[1,2,3,4]$. When metallic nanostructures are exposed to light at an appropriate wavelength free electrons in metallic nanostructures interact with light, and the collective oscillation of surface electrons leads to the surface plasmon resonance (SPR) [5, 6], which plays an important role for many applications. Obviously, how to fabricate nanostructured metallic films is a very important issue, and many techniques have been developed for that. Some of them are electron or ion beam lithography [7], wet chemical synthesis [8], inert gas condensation [9]. The other technique is annealing to transform structureless metallic films to the nanostructured metallic films through dewetting, and roughly speaking there are two types of annealing, i.e., thermal annealing and laser annealing techniques.

For thermal annealing [10] a film is heated to a certain temperature (but still below the melting point) by an electric furnace $[11,12,13]$, and atoms in the film diffuse to lower the surface energy. Such an atomic diffusion results in the formation of nanostructures, and this is so-called solid-state dewetting [14, 15]. Compared with the previously mentioned techniques, thermal annealing of thin metallic films is a very 
simple and inexpensive method to obtain nanostructures. The main drawback is that, including the heating and cooling time before and after reaching the target temperature, thermal annealing takes a few hours. A variant of thermal annealing is also known by replacing a conventional electric furnace by a special one for rapid thermal annealing (RTA) [16, 17, 18].

As for laser annealing [19] usually ultraviolet (UV) [19, 20, 21, 22] or occasionally mid-infrared lasers $[23,24,25]$ are employed to fabricate metallic nanostructures. By laser annealing in the UV wavelength the energy from a laser is absorbed by the film itself to instantly (within the pulse duration) heat it beyond the melting point to induce liquid-phase dewetting [26]. Although it works even for metallic films with high melting points, the drawback is that the laser beam has to be focused so that it can heat the film beyond the melting point, and accordingly the dimension of the nanostructured area is typically no more than a few hundred $\mu \mathrm{m}$. In contrast, if the laser is in the mid-infrared wavelength $(8 \sim 12 \mu \mathrm{m})$, the energy from the laser is mainly absorbed by the substrate which is typically made of glass or silicon. This means that a film deposited on the substrate is also heated, and annealing would take place $[23,24,25]$. $\mathrm{A} \mathrm{CO}_{2}$ laser is one of the most well-known mid-infrared lasers, and models with various laser powers (from a few $\mathrm{W}$ to tens of $\mathrm{kW}$ ) are commercially available. Because many of the $\mathrm{CO}_{2}$ lasers operate in the continuous wave mode or nearly continuous wave mode (i.e., pulse duration $\sim$ pulse interval), the substrate and hence a film on it is continuously heated during $\mathrm{CO}_{2}$ laser irradiation, so that the film can undergo dewetting at temperatures well below the melting point. That is, irradiation of $\mathrm{CO}_{2}$ laser in the nearly continuous wave mode can induce solid-state dewetting. Recently a focused $\mathrm{CO}_{2}$ laser beam has been employed to induce nanostructures in thin films [27, 28]. More recently we have demonstrated that the irradiation of a non-focused $\mathrm{CO}_{2}$ laser beam with a power density of $4 \sim 6 \mathrm{~W} / \mathrm{cm}^{2}$ to a $5 \mathrm{~nm} \mathrm{Au}$ film on a glass substrate for several seconds is sufficient to induce $\mathrm{Au}$ nanostructures [29]. Knowing that $\mathrm{CO}_{2}$ laser annealing of thin $\mathrm{Au}$ films works well, how it works for thin Ag films is an interesting question, since they are also frequently used for many applications. Note that the effect of $\mathrm{CO}_{2}$ laser annealing on thin Ag films is not necessarily the same with that on thin Au films, presumably because their melting points and masses are different: The melting points of bulk $\mathrm{Au}$ and $\mathrm{Ag}$ are 1064 and $962{ }^{\circ} \mathrm{C}$, while the masses of the respective atoms are 197 and 108 .

In this work we demonstrate that the wavelength of SPR of Ag NIs can be tuned quite a lot by the choice of the laser power and irradiation time. For instance, for the $5 \mathrm{~nm}$ Ag films prepared under the same sputtering condition, the resonance wavelength of $\mathrm{Ag}$ NIs can be tuned from $548 \mathrm{~nm}$ to $452 \mathrm{~nm}$ at the $3 \mathrm{~W}$ laser power simply by varying the irradiation time from 5 sec to $2 \mathrm{~min}$. Compared with the relatively simple and straightforward well-known technique to tune the wavelength of SPR of noble metal films through the deposition rate, film thickness, and substrate temperature [30], our technique offers a simple and quick alternative, since a nanostructured Ag film with a $\mathrm{SPR}$ at the desired wavelength can be fabricated on demand from the Ag films which have been prepared beforehand under the same sputtering condition. Perhaps more 
interestingly, the reduction of the height of SPR set in after the 30 sec irradiation at $3 \mathrm{~W}$ for $\mathrm{Ag}$ films and it almost disappears after the 10 min irradiation if the laser power is $5 \mathrm{~W}$, while it did not happen for Au films up to the 1 min irradiation even at $6 \mathrm{~W}$ [29]. The gradual loss of plasmon color, which means the disappearance of Ag NIs, at the irradiation center on the film implies that the diffusion of $\mathrm{Ag}$ atoms over the macroscopic distance occurs during the laser irradiation, and this interpretation is confirmed by the SEM analysis: Lesser and lesser Ag NIs are observed at the irradiation center as the irradiation time becomes longer.

\section{Experimental details}

We employ a cover glass (borosilicate glass, $18 \times 18 \times 0.15 \mathrm{~mm}$ ) for a microscope as a substrate. Before use we clean all substrates in the ultrasonic bath with deionised water for $5 \mathrm{~min}$. After that an Ag film with a $5 \mathrm{~nm}$ thickness is deposited on the substrate by magnetron sputtering (MSP-mini, Vacuum Device Ltd.) using an Ag target (purity $99.95 \%)$.

For laser annealing of $\mathrm{Ag}$ films we employ a $\mathrm{CO}_{2}$ laser at the wavelength of $10.6 \mu \mathrm{m}$ (AL30P, Access Laser Co., peak power $60 \mathrm{~W}$, pulse duration 100-400 $\mu$ s depending on the laser power, repetition rate $2.5 \mathrm{kHz}$ ). The laser beam has a nearly Gaussian transverse mode with $\mathrm{M}^{2} \sim 1.1$, and its diameter is $\sim 10 \mathrm{~mm}$ at the film position. To ensure the maximum absorption of laser energy by the glass substrate the laser is irradiated from the back side (i.e., glass side) of the film. In reality, however, whether the $\mathrm{CO}_{2}$ laser is irradiated from the front or back side of the film does not matter if the film thickness is $5 \mathrm{~nm}$, but it matters if it is beyond $\sim 7 \mathrm{~nm}$ [29]. The temperature of the Ag film during laser irradiation is measured using a thermocouple with a size of $\sim 1 \mathrm{~mm}$. To characterize the morphological and optical properties of the film we employ a scanning electron microscope (SEM) (JSM-6500FE, JEOL) with $10 \mathrm{kV}$ and a compact CCD spectrometer (USB2000+, Ocean Optics). Note that the SEM images and the optical absorption spectra shown in this work are obtained at the irradiation center of the film, unless otherwise noted. We fabricate Ag NI films several times under the same irradiation conditions to ensure that the reproducibility of Ag NIs is very good. Characterization of the fabricated films in terms of the number of particles, average diameter, density, and packing fraction has been performed by analyzing the SEM images of the Ag films with an open software, ImageJ v1.45.

\section{Results and discussions}

Figure 1(a) shows the SEM image of the as-deposited (0 W) Ag film with an enlarged view in the inset. As can be seen, the sputtered Ag films exhibit squashed island shapes (Volmer-Weber growth mode [31]). Figure 1(b) shows the SEM image of Ag NIs after the laser irradiation at $1 \mathrm{~W}$ for $5 \mathrm{sec}$. The applied laser power and/or irradiation time does 

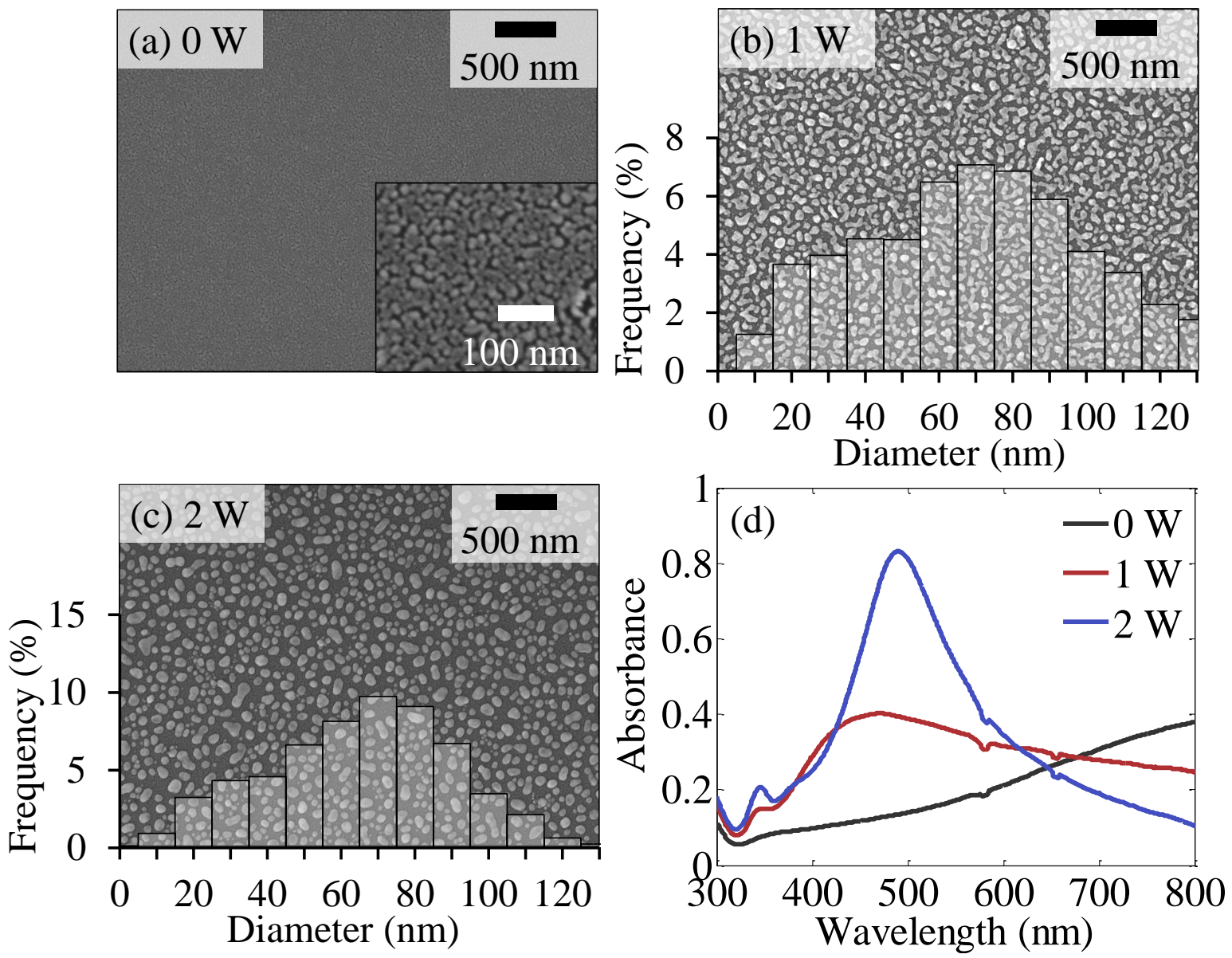

Figure 1: SEM images of Ag NIs after 5 sec laser irradiation at the laser powers of (a) $0 \mathrm{~W}$ (as-deposited), (b) $1 \mathrm{~W}$, and (c) $2 \mathrm{~W}$, and (d) optical absorption spectra.

not seem to be sufficient to induce the isolated NIs. When the laser power is increased to $2 \mathrm{~W}$, the temperature of the substrate and the film reaches $\sim 90{ }^{\circ} \mathrm{C}$ in 5 sec, and this laser power and irradiation time seems to be sufficient to form elongated NIs, as we see in figure 1(c). The optical absorption spectra of those films are summarized in figure $1(\mathrm{~d})$. Although the spectrum of the as-deposited Ag film does not show the SPR, that of the NIs at $1 \mathrm{~W}$ exhibits a broad SPR with a long tail on the long wavelength side, because the NIs are neither well-isolated nor very round. The spectrum at $2 \mathrm{~W}$ exhibits a much sharper SPR, because they are more round. Although tuning the surface plasmon resonance wavelength is possible by controlling the parameters of thermal evaporation deposition [30] it is difficult to obtain round nanoislands without an additional process such as laser/thermal annealing. As additional remarks regarding figure $\mathbf{1}(\mathbf{d})$ we note that the small peak at $\sim 350 \mathrm{~nm}$ must originate from the glass substrate itself, since the absorption spectrum of the laser-annealed substrate without an Ag film on it still shows the small peak at the same wavelength (not shown here). A small quadrupole resonance of $\mathrm{Ag}$ NIs is also visible in figure $1(\mathrm{~d})$ 

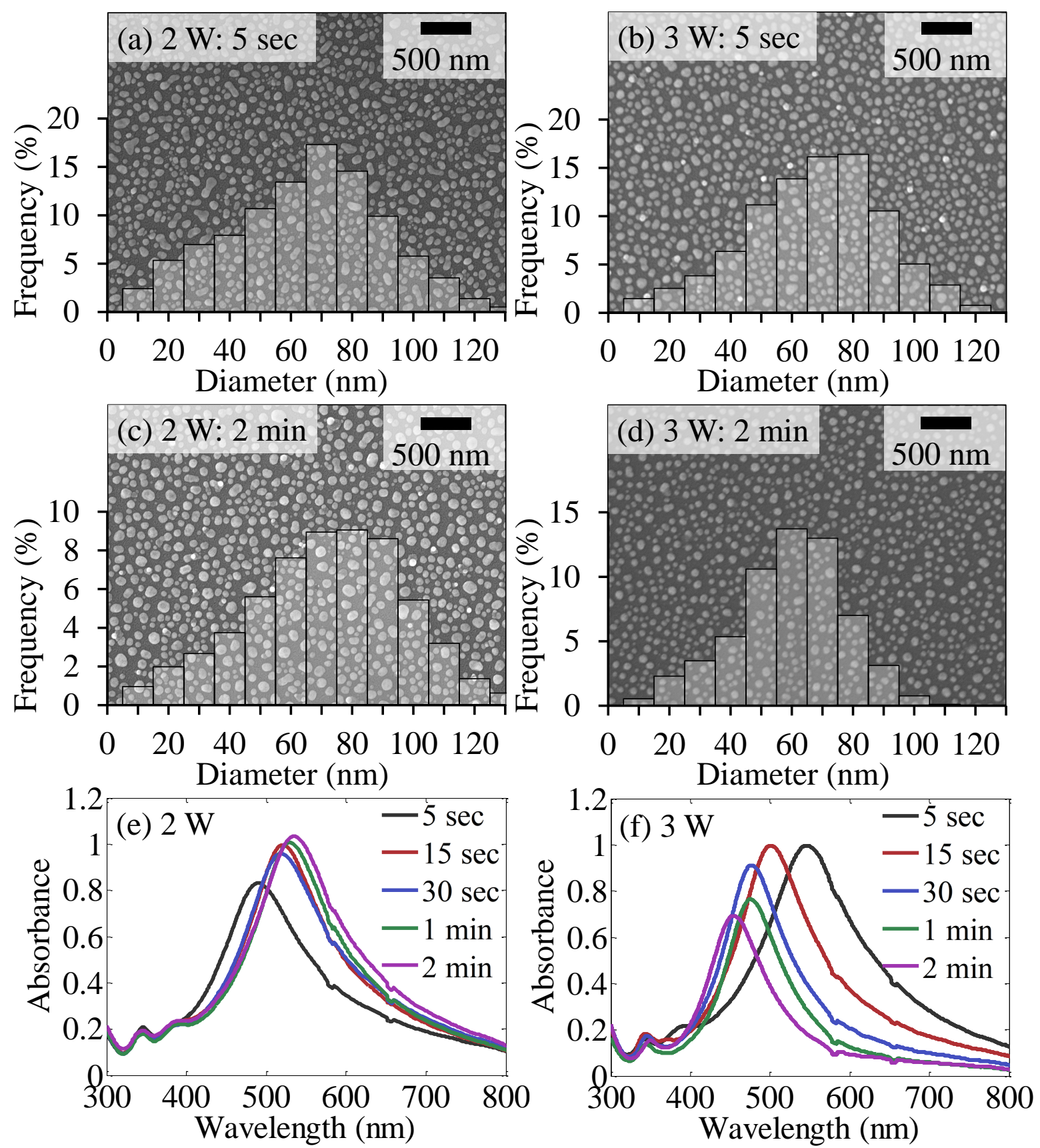

Figure 2: SEM images and size distributions of Ag NIs after laser irradiation at the laser powers and irradiation times of (a) $2 \mathrm{~W}$ for $5 \mathrm{sec}$, (b) $3 \mathrm{~W}$ for $5 \mathrm{sec}$, (c) $2 \mathrm{~W}$ for 2 min, and (d) $3 \mathrm{~W}$ for 2 min. Optical absorption spectra of Ag NIs after laser annealing at the laser powers of (e) $2 \mathrm{~W}$ and (f) $3 \mathrm{~W}$.

at around $390 \mathrm{~nm}$.

From figure 1(d) we have learned that, for the irradiation time of 5 sec, at least a laser power of $2 \mathrm{~W}$ is necessary to induce NIs with a clear SPR. Now, we perform the laser irradiation to the Ag films at 2 and $3 \mathrm{~W}$ for the irradiation times of $5 \mathrm{sec}$ and 
2 min. Figure 2 shows the SEM images together with the size distribution of NIs and the optical absorption spectra. In the case of laser irradiation for $5 \mathrm{sec}$ the use of higher laser power results in the larger size of NIs (compare figures 2(a) and (b)). In the case of longer irradiation time, i.e., $2 \mathrm{~min}$, the size distributions of NIs formed at the laser powers of 2 and $3 \mathrm{~W}$ turn out to be other way around, i.e., the use of higher laser power results in the smaller size of NIs. The optical absorption spectra of NIs obtained under those conditions are presented in figures $2(\mathrm{e})$ and (f) for the employed laser power of 2 and $3 \mathrm{~W}$, respectively. Those absorption spectra are consistent with the SEM images shown in figures 2(a)-(d): when the employed laser power is $2 \mathrm{~W}$ the peak position of SPR shifts to the longer wavelength side for the longer irradiation time, since the average diameter of NIs becomes larger. In contrast, when the employed laser power is $3 \mathrm{~W}$ the peak position of SPR shifts to the shorter wavelength side for the longer irradiation time, since the average diameter of NIs becomes smaller. We point out that a similar trend has been reported by others [32]. More interestingly, the height of the SPR at $3 \mathrm{~W}$ becomes smaller for the longer irradiation time (i.e., $30 \mathrm{sec}-2 \mathrm{~min}$ ), and this implies that a non-negligible mass transport of Ag atoms from the irradiation center to the peripheral region already set in at this laser power. This phenomenon becomes more prominent at the higher laser power, and we will make more discussions later on in this paper.

Since we have found that the laser power and the irradiation time on Ag films significantly affect the properties of NIs, we now carry out the comprehensive study on the morphological and optical properties of NIs. They are summarized in figure 3 together with the temporal variation of film temperature. First, the time evolution of film temperature is shown in figure 3(a) for the case in which the laser is suddenly turned on and off at time 0 and $120 \mathrm{sec}$, respectively. For all laser powers the film temperature rapidly increases and reaches the steady-state temperature during the first $30 \mathrm{sec}$, and then goes down to the room temperature in $1 \mathrm{~min}$ when the laser is suddenly turned off. Figures 3(b)-(d) shows the variation of the average diameter, number density, and packing fraction of induced NIs, defined by (total area of NIs)/(area of SEM image), as a function of time. Although the behavior of the number density of NIs (figure 3(c)) is a bit irregular, in particular for the first $30 \mathrm{sec}$, the behaviors of all those three quantities may be roughly divided into two groups in terms of the laser power: low laser power (1-3 W) and high laser power (4-6 W) groups. For instance, after the 2 min irradiation the average diameter of NIs is relatively large $(55-70 \mathrm{~nm})$ when the laser power is low, while it is smaller (17-34 nm) when the laser power is high. It is interesting to point out that the variation of the average diameter for the case of $4 \mathrm{~W}$ (figure $3(\mathrm{~b})$ ) reveals a sharp drop from 70 to $35 \mathrm{~nm}$ during the first $30 \mathrm{sec}$, after which it stays nearly the same. This behavior seems to lie between those of low and high laser power groups. A possible reason for this is that the diffusion speeds of Ag atoms/clusters/NIs on the substrate increases at the higher film temperature [33] so that some of NIs under the laser irradiation at $4 \mathrm{~W}$ may have moved out of the irradiation center. It is not likely that the NIs melt at this laser power, since the film temperature at $4 \mathrm{~W}$ cannot be 
Surface plasmon resonance tuning of Ag nanoisland films using a $\mathrm{CO}_{2}$ laser
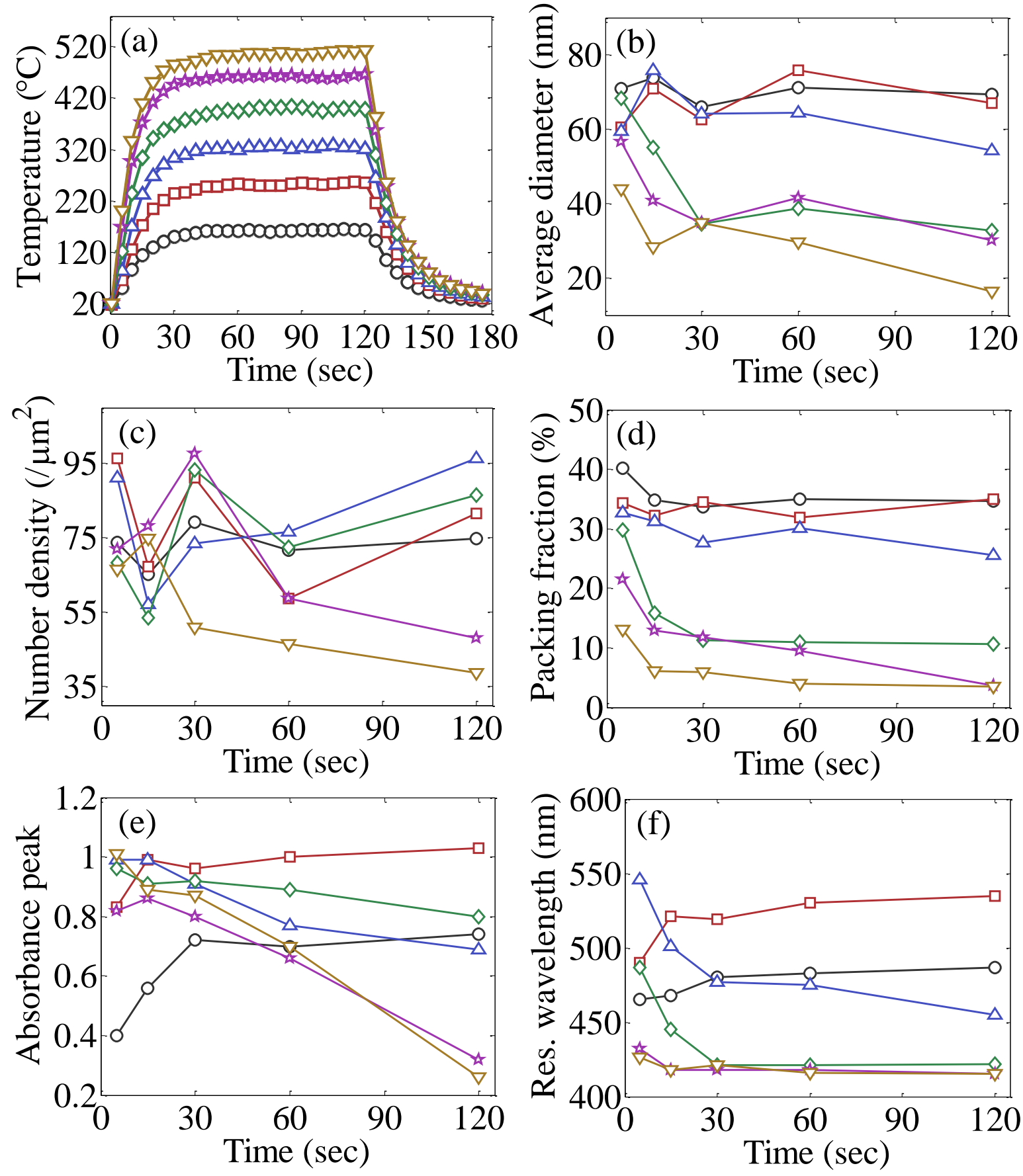

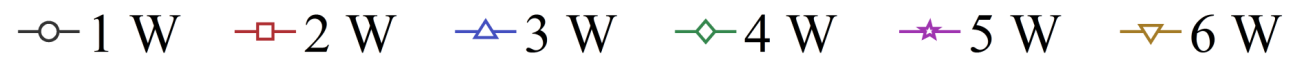

Figure 3: Variation of the (a) film temperature and (b) average diameter, (c) number density, (d) packing fraction, (e) height of the SPR peak, and (f) wavelength of SPR of $\mathrm{Ag}$ NIs as a function of laser irradiation time at the different laser powers $(1,2,3,4$, and $5 \mathrm{~W})$. 
higher than $400{ }^{\circ} \mathrm{C}$ (figure 3(a)) and the melting point of $\mathrm{Ag}$ NIs cannot be lower than $90 \%$ of that of bulk Ag [34]. The behavior of packing fraction (figure 3(d)) is simple and clear at any laser power. At a low laser power (1-3 W) the packing fraction hardly changes, while at a high laser power (4-6 W) it rapidly decreases during the first 30 sec and then remains nearly the same. These results also seem to suggest that some amount of NIs have moved out of the observed area.

Now we turn to the optical properties of NIs. Figures 3(e) and (f) show the height and resonance wavelength of SPR as a function of time. The behaviors of the height of SPR (figure 3(e)) do not show any clear trend, and therefore we look into the respective result at the different laser power: At $1 \mathrm{~W}$ the height of SPR increases for the longer irradiation time without so much change in the number density or packing fraction. This means that the NIs change their shapes from the irregular or elongated ones to the more round ones without coalescence or break up. At 2-3 W the laser power is sufficient to induce NIs during the first several seconds, and then they have ripened so that little morphological change occurs. This is why the height of SPR remains nearly the same. At $4 \mathrm{~W}$, however, the movement of NIs out of the irradiation center becomes faster and hence non-negligible due to the faster diffusion speed of NIs at the higher film temperature. At $5 \mathrm{~W}$, more NIs move out of the irradiation center. Accordingly, the height of SPR decreases down to $\sim 0.3$ in 120 sec (figure 3(e)). Finally, the behavior of the resonance wavelength (figure $3(\mathrm{f})$ ) essentially reflects the behavior of the average diameter of NIs shown in figure 3(b). Recall that the similar phenomenon was not observed in $5 \mathrm{~nm} \mathrm{Au}$ films at least up to 1 min irradiation at $5 \mathrm{~W}$, and even after the 5 min irradiation at $5 \mathrm{~W}$ the reduction of the height of SPR was very little [29].

If the laser irradiation time is chosen to be much longer we can more clearly see the interesting behavior of NIs mentioned above. Figures 4(a)-(c) show the SEM images of NIs obtained by the 10 min irradiation at the laser powers of 1,3 , and $5 \mathrm{~W}$, respectively. It is interesting to point out that the properties of NIs after the 10 min irradiation is very different by the choice of the laser power: When the laser power is $1 \mathrm{~W}$, the NIs, which are not well-isolated after the $5 \mathrm{sec}$ irradiation (figure 1(b)), become much better-isolated after the $10 \mathrm{~min}$ irradiation without so much change in the number density (figure 4(a)). This morphological change (figure 1(b) $\rightarrow$ figure 4(a)) is consistent with the change in the optical absorption spectra (figure $1(\mathrm{~d}) \rightarrow$ figure $4(\mathrm{~d})$ ). When the laser power is $3 \mathrm{~W}$, the SEM images of NIs after the $5 \mathrm{sec}, 2 \mathrm{~min}$, and $10 \mathrm{~min}$ irradiation (figure $2(\mathrm{~b}) \rightarrow$ figure $2(\mathrm{~d}) \rightarrow$ figure $4(\mathrm{~b})$ ) are somehow different, and the average diameter of NIs is smaller after the longer irradiation time. This fact is also consistent with the optical absorption spectra shown in figure 2(f) and figure 4(d). When the laser power is $5 \mathrm{~W}$, however, we hardly see NIs after the $10 \mathrm{~min}$ irradiation (figure 4(c)). This does not mean that there are many small NIs which are not visible by SEM, since, if so, a clear SPR must appear in the optical absorption spectrum. The fact is that, we see a very small SPR at $\sim 410 \mathrm{~nm}$, as shown in figure $4(\mathrm{~d})$. Obviously, the small SPR at such a short wavelength arises from the contribution of some small NIs which are not visible by our SEM. Therefore, the disappearance of NIs after the 10 min irradiation at $5 \mathrm{~W}$ 
implies that most of the NIs have moved out of the irradiation center to the peripheral region.

To confirm the above interpretation it is necessary to clarify how the spatial profile of the laser beam influences the morphological and optical properties of NIs induced at different positions on the Ag films. For this purpose we take the SEM images at different positions with respect to the irradiation center. The results are summarized in figures $5(\mathrm{a})$-(e) for the case of $10 \mathrm{~min}$ irradiation at $5 \mathrm{~W}$. Figure $5(\mathrm{f})$ shows the photo of the entire $(18 \mathrm{~mm} \times 18 \mathrm{~mm}$ size $)$ Ag film after the laser irradiation. As we notice the central part with a few mm diameter is nearly transparent (figure 5(f)) with a very small SPR (figure 6(b)), implying that most of the NIs have moved out of the irradiation center to the outer region to leave the irradiation center with very few Ag NIs (figure 5(a)). Moreover, the disappearance of Ag NIs at the irradiation center is not due to the jumping of nanoparticles [35] because the deformation speed of NIs on the substrate during solid-state dewetting is very slow. This is consistent with the SEM images and the optical absorption
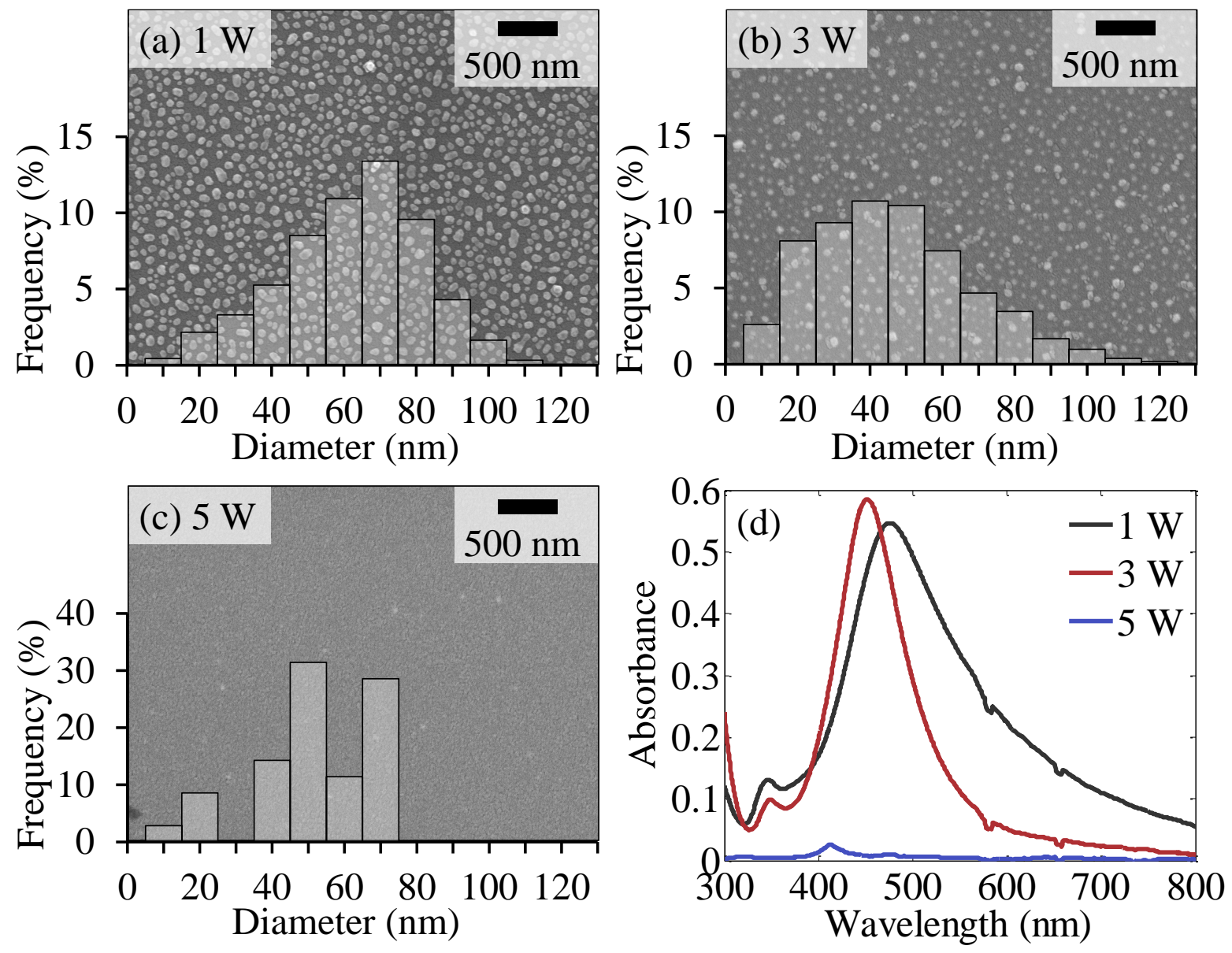

Figure 4: SEM images of Ag NIs after the 10 min laser iradiation at the laser powers of (a) $1 \mathrm{~W}$, (b) $3 \mathrm{~W}$, and (c) $5 \mathrm{~W}$, and (d) optical absorption spectra. 
spectrum at the irradiation center (i.e., $0 \mathrm{~mm}$ ) shown in figures $4(\mathrm{c})$ and (d) (for $5 \mathrm{~W}$ ). However, at the further position from the irradiation center more NIs are observed (figure $5(\mathrm{a}) \rightarrow$ figure $5(\mathrm{~b}) \rightarrow$ figure 5 (c) corresponding to $0 \mathrm{~mm} \rightarrow 3 \mathrm{~mm} \rightarrow 4 \mathrm{~mm}$ ). By going even further away from the irradiation center (figure $5(\mathrm{~d}) \rightarrow$ figure $5(\mathrm{e})$ corresponding to $5 \mathrm{~mm} \rightarrow 6 \mathrm{~mm}$ ) the NIs exhibit more irregular and elongated shapes compared with those at $4 \mathrm{~mm}$ (figure $5(\mathrm{c})$ ). Clearly, this is reminiscent of the NIs induced by the laser irradiation at the low laser power (figures 1). Interesting that the

The number density and packing fraction of Ag NIs as well as the optical absorption spectra at the different positions from the irradiation center are presented in figures 6(a) and (b), respectively, under the same irradiation condition employed for figure 5 . Figure 6(b) clearly shows that, at the laser power of $5 \mathrm{~W}$ and the irradiation time of $10 \mathrm{~min}$, the distance of $4 \mathrm{~mm}$ from the irradiation center is most appropriate to obtain more homogeneous Ag NIs with the highest packing fraction.

Before closing this section we emphasize that the macroscopic diffusion of Ag atoms does not require the laser power of $5 \mathrm{~W}$ and long irradiation time. Figure 7 demonstrates that it occurs even at the laser power of $3 \mathrm{~W}$ within $30 \mathrm{sec}$, and by referring to Figure 3(a) the film temperature does not exceed $320^{\circ} \mathrm{C}$ under this irradiation condition. Of course, if the solid-state dewetting is induced by thermal annealing with an electric furnace this kind of atomic diffusion would not take place since there is no temperature gradient on the substrate. However, similar phenomena would be observed if laser annealing with
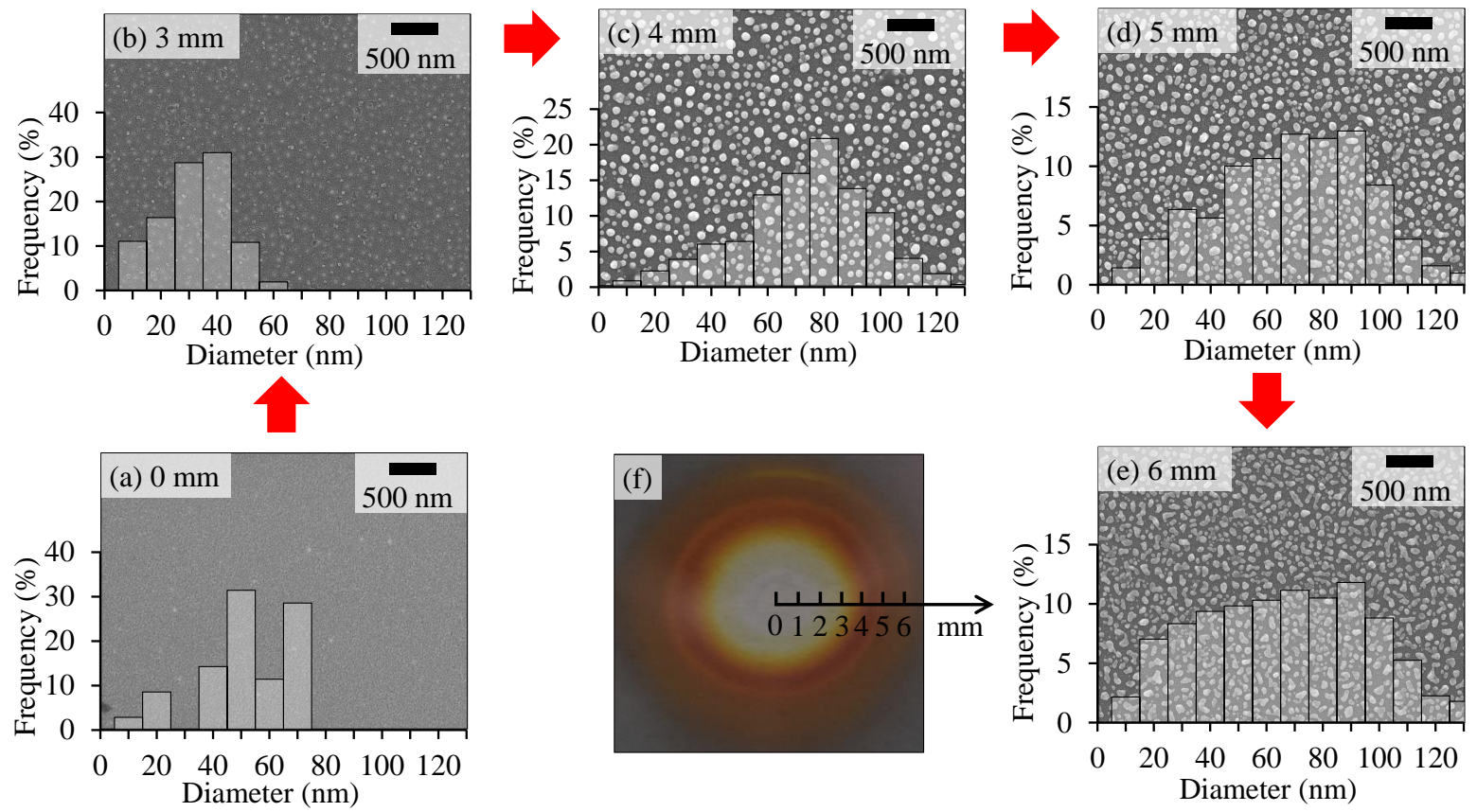

Figure 5: SEM images and size distributions of $\mathrm{Ag}$ NIs at (a) $0 \mathrm{~mm}$, (b) $3 \mathrm{~mm}$, (c) $4 \mathrm{~mm}$, and (d) $5 \mathrm{~mm}$, and (e) $6 \mathrm{~mm}$ from the center of the irradiated area. Laser power and irradiation time are $5 \mathrm{~W}$ and $10 \mathrm{~min}$ for all (a)-(e). (f) Photo of the irradiated film. 
a focused UV laser is employed to induce NIs. To our knowledge there is no such a report in the literature. One possible reason for this would be that, although the similar effect is perhaps there by UV laser annealing, it may be smeared out after scanning the irradiated spot (typically a few tens of $\mu \mathrm{m}$ ) on the film to make the area of NIs to a few mm or larger. Another possible reason for this is that, if a single laser pulse, typically with a fs $\sim$ ns duration, is employed to induce nanostructures, the time duration for the atoms in the film to be at the sufficiently high temperature is too short to move over a sufficient distance. To observe the visible color as well as structural change in the target film multiple irradiation onto the same spot would be necessary. In contrast our $\mathrm{CO}_{2}$ laser is nearly continuous wave since the pulse duration is comparable to the pulse interval, and the atoms in the film have sufficient time to move on the substrate. An interesting application of Ag NIs films with the radial plasmonic color change as shown in figure 7 is to use them for SERS (surface enhanced raman scattering) substrates: Since the broad range of resonant wavelengths is realized on a single film different pump laser wavelengths can be used for a sample on a single Ag NIs film only if the irradiation spot is appropriately chosen.

\section{Conclusions}

In conclusion, we have demonstrated that the surface plasmon resonance of Ag NIs can be tuned over the wide wavelength range through the laser power and irradiation time. Interestingly, we have observed the gradual loss of plasmon color at the irradiation center during the irradiation, and from the position-dependent optical absorption spectra and SEM analysis we have confirmed that the diffusion of $\mathrm{Ag}$ atoms from the irradiation
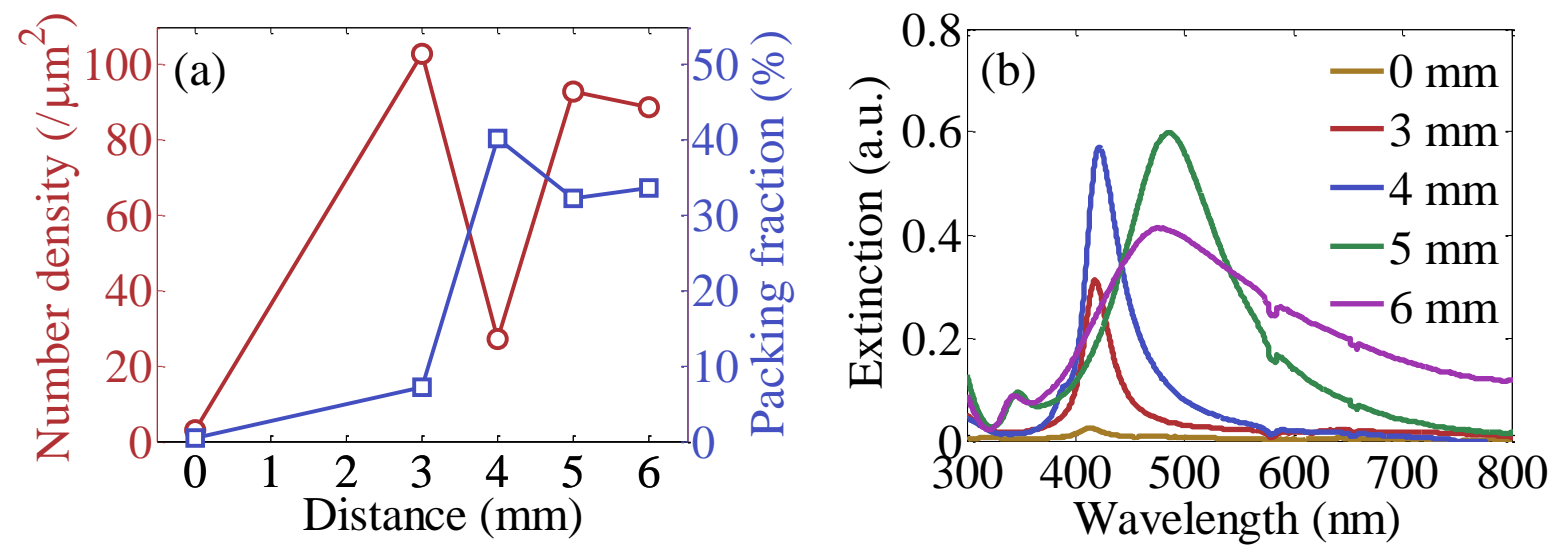

Figure 6: (a) Number density (left axis) and packing fraction (right axis) of Ag NIs as a function of distance from the center of the irradiated area. (b) Optical absorption spectra of $\mathrm{Ag}$ NIs at the different positions (0,3,4,5, and $6 \mathrm{~mm}$ ) from the center of irradiated area. The laser power and irradiation time are $5 \mathrm{~W}$ and $10 \mathrm{~min}$, respectively, as employed for figure 5 . 

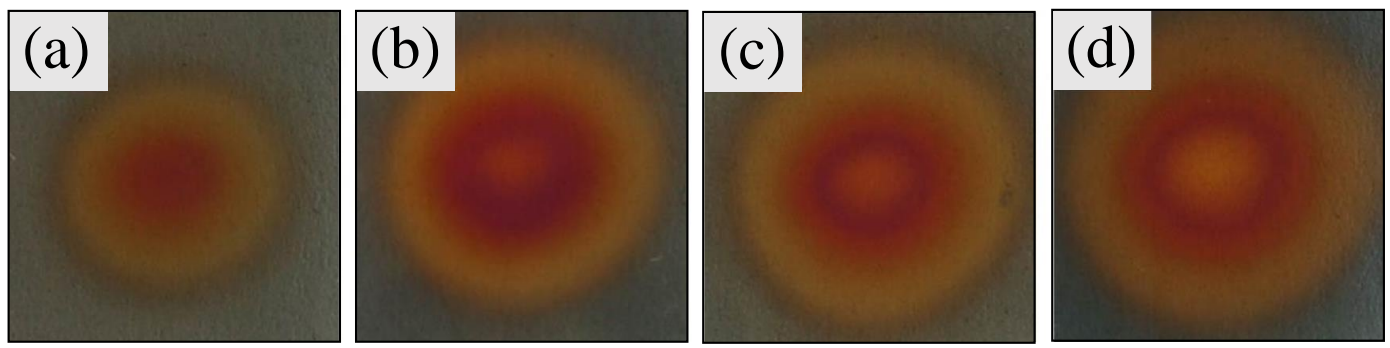

Figure 7: Color change of $\mathrm{Ag}$ NI films after the $\mathrm{CO}_{2}$ laser irradiation at $3 \mathrm{~W}$ for the irradiation times of (a) 5, (b) 10, (c) 15, and (d) 30 sec.

center to the peripheral region takes place. The temperature gradient of the substrate due to the spatial profile of the laser beam is the driving force for this. Our results imply that we are able to conveniently fabricate Ag NI films with radial-position-varying optical and morphological properties on a single film, which can be useful, for instance, for SERS applications.

\section{Acknowledgements}

This work was supported by a Grant-in-Aid for Scientific Research from the Ministry of Education, Culture, Sports, Science and Technology of Japan.

\section{References}

[1] Bruchez M, Moronne M, Gin P, Weiss S and Alivisatos A P 1998 Semiconductor nanocrystal as fluorescent biological labels Science $\mathbf{2 8 1} 2013$

[2] Wang S, Mamedova N, Kotov N A, Chen W and Studer J 2002 Antigen/antibody immunocomplex from CdTe nanoparticle bioconjugate Nano Lett. 2817

[3] Abdulhalim I 2014 Plasmonic sensing using metallic nanosculptured thin films Small 103499

[4] Sannomiya T, Scholder O, Jefimovs K, Hafner C and Dahlin A B 2011 Investigation of plasmon resonances in metal films with nanohole arrays for biosensing applications Small 71653

[5] Willets K A and Van Duyne R P 2007 Localized surface plasmon resonance spectroscopy and sensing Annu. Rev. Phys. Chem. 58267

[6] Mayer K M and Hafner J H 2011 Localized surface plasmon resonance sensors Chem. Rev. 111 3828

[7] Lindquist N C, Nagpal P, McPeak K M, Norris D J and Oh S-H 2012 Engineering metallic nanostructures for plasmonics and nanophotonics Rep. Prog. Phys. 75036501

[8] Jana N R, Gearheart L and Murphy C J 2001 Wet chemical synthesis of silver nanorods and nanowires of controllable aspect ratio Chem. Comm. 7617

[9] Baker C, Pradhan A, Pakstis L, Pochan D J and Shah S I 2005 Synthesis and antibacterial properties of silver nanoparticles J. of Nanosc. and Nanotech. 5244

[10] Anker J N, Hall W P, Lyandres O, Shah N C, Zhao J and Van Duyne R P 2008 Biosensing with plasmonic nanosensors Nature Materials 7442

[11] Sun H, Yu M, Wang G, Sun X and Lian J 2012 Temperature-dependent morphology evolution and surface plasmon absorption of ultrathin gold island films J. Phys. Chem. C 1169000

[12] Sun X and Li H 2013 Gold nanoisland arrays by repeated deposition and post-deposition annealing for surface-enhanced Raman spectroscopy Nanotechnology 24355706 
[13] Kracker M, Worsch C, Bocker C and Rssel C 2013 Optical properties of dewetted thin silver/gold multilayer films on glass substrates Thin Solid Films $\mathbf{5 3 9} 47$

[14] Thompson C V 2012 Solid-state dewetting of thin films Annu. Rev. Mater. Res. 42399

[15] Leroy F, Borowik L, Cheynis F, Almadori Y, Curiotto S, Trautmann M, Barbé J C and Müller P 2016 How to control solid state dewetting: a short review Surf. Sc. Rep. $\mathbf{7 1} 391$

[16] Walter D C, Lim B, Bothe K, Voronkov V V, Falster R and Schmidt J 2014 Effect of rapid thermal annealing on recombination centres in boron-doped Czochralski-grown silicon Appl. Phys. Lett. 104042111

[17] Seguini G, Curi J L, Spiga S, Tallarida G, Wiemer C and Perego M 2014 Solid-state dewetting of ultra-thin $\mathrm{Au}$ films on $\mathrm{SiO}_{2}$ and $\mathrm{HfO}_{2}$ Nanotechnology 25495603

[18] Lin H T H, Yang C K, Lin C C, Wu A M H, Wang L A and Huang N T 2017 A large-area nanoplasmonic sensor fabricated by rapid thermal annealing treatment for label-free and multipoint immunoglobulin sensing Nanomaterials 7100

[19] Henley S J, Carey J D and Silva S R P 2005 Pulsed-laser-induced nanoscale island formation in thin metal-on-oxide films Phys. Rev. B 72195408

[20] Imamova S, Nedyalkov N, Dikovska A, Atanasov P, Sawczak M, Jendrzejewski R, Śliwiński G and Obara M 2010 Near field properties of nanoparticle arrays fabricated by laser annealing of thin Au and Ag films Appl. Surf. Sci. 2571075

[21] Baumgardner W J, Choi J J, Bian K, Kourkoutis L F, Smilgies D M, Thompson M O and Hanrath T 2011 Pulsed laser annealing of thin films of self-assembled nanocrystals ACS Nano 57010

[22] Fang Y, Hou Y, Teng F, Lou Z, Tang A, Zhang B, Meng L, Ning Y and Lu L 2013 Characterization of nanoscale clusters fabricated by pulsed laser irradiation of thin Au films Appl. Surf. Sci. $\mathbf{2 7 3}$ 625

[23] Tewary A, Kekatpure R D and Brongersma M L 2006 Controlling defect and Si nanoparticle luminescence from silicon oxynitride films with $\mathrm{CO}_{2}$ laser annealing Appl. Phys. Lett. 88093114

[24] Halabica A, Idrobo J C, Pantelides S T, Magruder R H, Pennycook S J and Haglund R F 2008 Pulsed infrared laser annealing of gold nanoparticles embedded in a silica matrix J. Appl. Phys. 103083545

[25] Nyga P, V. Drachev V P, Thoreson M D and Shalaev V M 2008 Mid-IR plasmonics and photomodification with Ag films Appl. Phys. B 9359

[26] Trice J, Thomas D, Favazza C, Sureshkumar R and Kalyanaraman R 2007 Pulsed-laser-induced dewetting in nanoscopic metal films: Theory and experiments Phys. Rev. B 75235439

[27] Ooms M D, Jeyaram Y and Sinton D 2015 Disposable plasmonics: rapid and inexpensive large area patterning of plasmonic structures with $\mathrm{CO}_{2}$ laser annealing Langmuir 315252

[28] Chung C K, Li C H and Chen T S 2015 Synthesis and characteristics of nanostructured siliconrich nitride thin films using modified long-wavelength $\mathrm{CO}_{2}$ laser annealing Laser Phys. Lett. 12 096003

[29] Maurya S K, Uto Y, Kashihara K, Yonekura N and Nakajima T 2018 Rapid formation of nanostructures in Au films using a $\mathrm{CO}_{2}$ laser Appl. Surf. Sci. 427961

[30] Weimer W A and Dyer M J 2001 Tunable surface plasmon resonance silver films Appl. Phys. Lett. 793164

[31] Venables J 1984 Nucleation and growth of thin films Rep. Prog. Phys. 47399

[32] Bhattacharyya S R, Datta D, Shyjumon I, Smirnov B M, Chini T K, Ghose D and Hippler R 2009 Growth and melting of silicon supported silver nanocluster films J. Phys. D: Appl. Phys. 42 035306

[33] Quan J, Zhang J, Qi X, Li J, Wang N and Zhu Y 2017 A study on the correlation between the dewetting temperature of Ag film and SERS intensity Sci. Rep. 714771

[34] Nanda K K, Sahu S N and Behera S N 2002 Liquid-drop model for the size-dependent melting of low-dimensional systems Phys. Rev. A 6690

[35] Habenicht A, Olapinski M, Burmeister F, Leiderer P and Boneberg J 2005 Jumping nanodroplets Science 3092043 